\title{
COMPARTILHAMENTO DO CONHECIMENTO EM SITUAÇÕES DE CRISES: REVISÃO SISTEMÁTICA DA LITERATURA
}

\author{
KNOWLEDGE SHARING IN CRISIS SITUATIONS: A \\ SYSTEMATIC LITERATURE REVIEW
}

\author{
João Augusto Wendt Mischiatti a \\ Helena de Fátima Nunes Silva ${ }^{\mathrm{b}}$ \\ Maria Gabriela Reis Carvalho ${ }^{c}$
}

\begin{abstract}
RESUMO
Objetivo: Apresentar uma revisão sistemática da literatura que investiga a forma de aplicação do compartilhamento do conhecimento em situações de crises nas organizações dos últimos quatro anos. Metodologia: Revisão Sistemática da Literatura conduzida pelo método PRISMA, com seleção de 24 artigos científicos, compreendendo o período de janeiro de 2017 até maio de 2021. Os artigos foram divididos em oito categorias: i) confiança; ii) memória institucional; iii) diversidade nas organizações; iv) efeitos da crise no compartilhamento do conhecimento; v) ferramentas para compartilhamento do conhecimento; vi) técnicas e estratégias para o compartilhamento do conhecimento; e vii) barreiras para o compartilhamento do conhecimento. Resultados: Os resultados constataram uma heterogeneidade dos estudos quanto às suas características, porém verificou uma predominância nos artigos do compartilhamento do conhecimento em épocas de crises referente às técnicas e estratégias (45\% do total) e nas ferramentas para compartilhamento do conhecimento ( $25 \%$ do total). Além disso, os estudos destacaram a importância de utilizar as melhores ferramentas, práticas e estratégicas de compartilhamento do conhecimento em situações de crise. Conclusões: Foi possível perceber a crescente importância desse tema nos últimos anos devido à pandemia da COVID-19. Verificou-se a necessidade de estudos futuros que possam identificar as principais estratégias de compartilhamento do conhecimento para enfrentar situações de crises.
\end{abstract}

Descritores: Compartilhamento de conhecimento. Conhecimento nas organizações. Gestão do conhecimento. Revisão de literatura.

\footnotetext{
a Doutorando em Gestão da Informação pela Universidade Federal do Paraná (UFPR). Analista de Gestão em Saúde da Fiocruz. E-mail: joaomischiatti@hotmail.com

b Doutora em Engenharia de Produção pela Universidade Federal de Santa Catarina (UFSC). Docente do Programa de Pós-Graduação em Ciência, Gestão e Tecnologia da Informação, da Universidade Federal do Paraná (UFPR). E-mail: helenanunes@gmail.com

c Doutora Engenharia Mecânica pela Universidade Federal de Minas Gerais (UFMG). Pesquisadora Colaboradora do Programa de Pós-Graduação em Engenharia Mecânica e de Materiais da Universidade Tecnológica Federal do Paraná (UTFPR). E-mail: carvalho.mariagabriela@gmail.com
} 


\section{INTRODUÇÃO}

As economias nacionais e mundiais - bem como suas organizações e sociedades componentes - têm presenciado nos últimos anos vários tipos de crises, desde escalas regionais até globais (WENZEL; STANKE; LIEBERMAN, 2020). As crises diferem em suas origens e proporções, porém, em sua maioria, elas possuem potencial de dano social, econômico e ambiental que pode ser devastador na localidade atingida. Muitas vezes os efeitos são esmagadores a ponto de se tornarem transfronteiriços e até mesmo globais (BANSAL; KIM; WOOD, 2018; WENZEL; STANKE; LIEBERMAN, 2020).

Observa-se que as organizações, a sociedade e os indivíduos apresentam maior vulnerabilidade emocional durante as situações de crise ou emergência, sendo comum a redução da eficiência operacional ou conclusão prejudicada de tarefas essenciais. Em tais cenários, o compartilhamento do conhecimento desempenha papel fundamental, pois a desorganização e a desestruturação impostas pela crise prejudicam a coleta e processamento de dados, com impacto em toda cadeia laboral ou produtiva (DICKMANN et al., 2014; GRUNNAN; FRIDHEIM, 2017). Assim, impõe-se como necessário analisar quais são as melhores práticas para que um efetivo compartilhamento de conhecimento seja utilizado da melhor forma na gestão de crises.

Para mitigar os efeitos dessas crises causadas por diversos motivos (desastres naturais, bolhas financeiras, pandemias, problemas políticos, entre outros) é necessário por parte dos agentes envolvidos a formação de estratégias, de colaboração e de bons métodos de compartilhamento do conhecimento para que seja realizada uma gestão eficaz no combate às crises (DICKMANN et al., 2014; GRAY et al., 2017; ZHANG et al., 2017; ÇAKAR, 2018; HUANG; SHIHWEI, 2020).

Dessa forma, para que as organizações estejam mais preparadas para a crise, é necessária uma boa gestão do conhecimento. Este é um recurso valioso, considerado uma vantagem competitiva para as organizações uma vez que aumenta conforme sua utilização, além de ser difícil de ser imitado (GRANT, 1996; DAVENPORT; PRUSAK, 1998; FOSS; PEDERSEN, 2002; PERSSON; 
STIRNA; AGGESTAM, 2008).

Assim, a Gestão do Conhecimento (GC), que é o processo pelo qual uma instituição facilita o compartilhamento do conhecimento de forma sistemática (MACHADO; ELIAS, 2020), surge como apoio fundamental para a organização, sendo necessária para a geração, o armazenamento e o compartilhamento do conhecimento (ALVARENGA NETO, 2008; ASRAR-UL-HAQ; ANWAR, 2016). Já o compartilhamento do conhecimento é o intercâmbio de conhecimentos tácitos e explícitos entre indivíduos, com o objetivo de colaborar com outros agentes para que seja resolvido um problema, criar novas ideias ou tornar este conhecimento acessível a outros membros (GROTTO, 2003; CUMMINGS, 2004).

O compartilhamento do conhecimento, quando utilizado adequadamente, provoca um aumento no desempenho organizacional tanto na medida tangível quanto intangível (ALI; PARIS; GUNASEKARAN, 2019). Pesquisas anteriores mostraram os benefícios do compartilhamento do conhecimento, que se traduzem na redução de custos, nos ciclos mais curtos de desenvolvimento de produtos, e em maior capacidade de inovação e desempenho (AHMAD; KARIM, 2019).

Instituições e governos lidam com uma ampla gama de crises em um ambiente cada vez mais complexo (GRUNNAN; FRIDHEIM, 2017). Nessas novas formas de crises há um cenário de incerteza e mudança, cabendo aos gestores de riscos proporem respostas inovadoras e rápidas empregando as informações e conhecimentos disponíveis no momento. (GRUNNAN; FRIDHEIM, 2017). Frente à relevância do tema, a questão de pesquisa do presente estudo é: Como ocorre o compartilhamento do conhecimento em situações de crises?

Respondendo a esse questionamento, o presente estudo tem como objetivo realizar uma revisão sistemática da literatura dos últimos quatro anos para apresentar as principais formas de compartilhamento do conhecimento aplicado em situações de crises, questionando quais são as práticas que favorecem a interação entre as organizações para que haja desenvolvimento de um ambiente propício ao compartilhamento do conhecimento. Também são 
elencados facilitadores, barreiras e estratégias encontrados nas instituições para criação e compartilhamento do conhecimento no enfrentamento de crises.

\section{METODOLOGIA}

Este artigo tem uma abordagem qualitativa de revisão sistemática da literatura, que envolve critérios rigorosos de uma busca abrangente e imparcial (TRANFIELD; DENYERD; SMART, 2003; CRONIN; RYAN; COUGHLAN, 2008; DIAS; SADAO; VILAS, 2019). Ele foi desenvolvido segundo as diretrizes e recomendações de Moher et al. (2009), pertinentes ao método Preferred Reporting Items for Systematic Reviews and Meta-Analyses (PRISMA). A abordagem qualitativa é complementada por alguns aspectos quantitativos, principalmente no que se refere à segmentação e agrupamento temático dos resultados, evidenciando o efeito temporal da pandemia da COVID-19 sobre a produção científica no período considerado.

Uma revisão sistemática é um método que busca avaliar, identificar e analisar os principais resultados de pesquisas já realizadas referentes a um determinado tópico (WOLOR et al., 2020). Nas revisões sistemáticas qualitativas as etapas a serem realizadas são: 1) formulação de perguntas de pesquisa; 2) realização de revisões sistemáticas de literatura; 3) triagem e seleção de artigos; 4) realização de análise e síntese de resultados qualitativos; 5) implementação de controle de qualidade; 6) preparação de um relatório final (PERRY; HAMMOND, 2002; WOLOR et al., 2020).

O estudo foi realizado com busca de publicações disponíveis nas bases de dados Scopus (https://www.scopus.com) e Web of Science (https://webofknowledge.com). A escolha da Web of Science deve-se à sua extensa cobertura temática e quantitativa, posto que essa base possui mais de 12 mil revistas cadastradas, contando com artigos indexados por revistas que possuem ISI (International Scientific Information), e é considerada a base de dados mais conhecida internacionalmente (FAHIMNIA; SARKIS; DAVARZANI, 2015; MISCHIATTI; MOLETA; CATAPAN, 2017). A base Scopus foi escolhida por possuir dezenas de milhões de artigos revisados por pares (FAHIMNIA; SARKIS; DAVARZANI, 2015; MISCHIATTI; MOLETA; 
CATAPAN, 2017), e é o maior banco de dados de resumos e citações da literatura na pesquisa.

O segundo passo foi definir as palavras-chave, as quais precisam ser selecionadas com cuidado para obter o melhor resultado possível (CRONIN; RYAN; COUGHLAN, 2008). Para levantamento dos termos elegíveis foi realizada leitura de teses, dissertações e artigos sobre o tema "compartilhamento do conhecimento". Também foi executada busca de thesaurus na EBSCO (https://www.ebsco.com) para verificar termos similares e úteis para a pesquisa. Assim foram definidos os seguintes termos para a estratégia de buscas nas bases de dados mencionadas acima: ("knowledge sharing" OR "knowledge transfer*") AND (crisis). Não foram realizadas buscas com os termos em português. Apesar de não restringir a nenhum idioma, só foram encontrados artigos em inglês sobre $o$ assunto.

Importante salientar que devido ao fato de muitos autores utilizarem os termos "knowledge sharing" e "knowledge transfer" como expressões intercambiáveis (PAULIN; SUNESON, 2012; TANGARAJA et al., 2016), optou-se por ampliar a busca para que alcançasse todos os artigos relevantes sobre o tema e, assim, fosse possível obter uma resposta ao questionamento: Como ocorre o compartilhamento do conhecimento em situações de crises?

No mês de maio de 2021 foi realizada a busca nas bases de dados Scopus e Web of Science com limite de data de publicação de janeiro de 2017 até maio de 2021. Embora o horizonte definido para a revisão fosse de quatro anos - portanto até janeiro de 2021 - os autores optaram por estender os resultados até o mês de maio, a fim cobrir a defasagem natural entre a publicação dos artigos e sua indexação pelas diferentes bases de dados. A Figura 1 representa o fluxograma de identificação e seleção dos artigos localizados. 
Figura 1 - Fluxograma de seleção dos estudos (PRISMA)

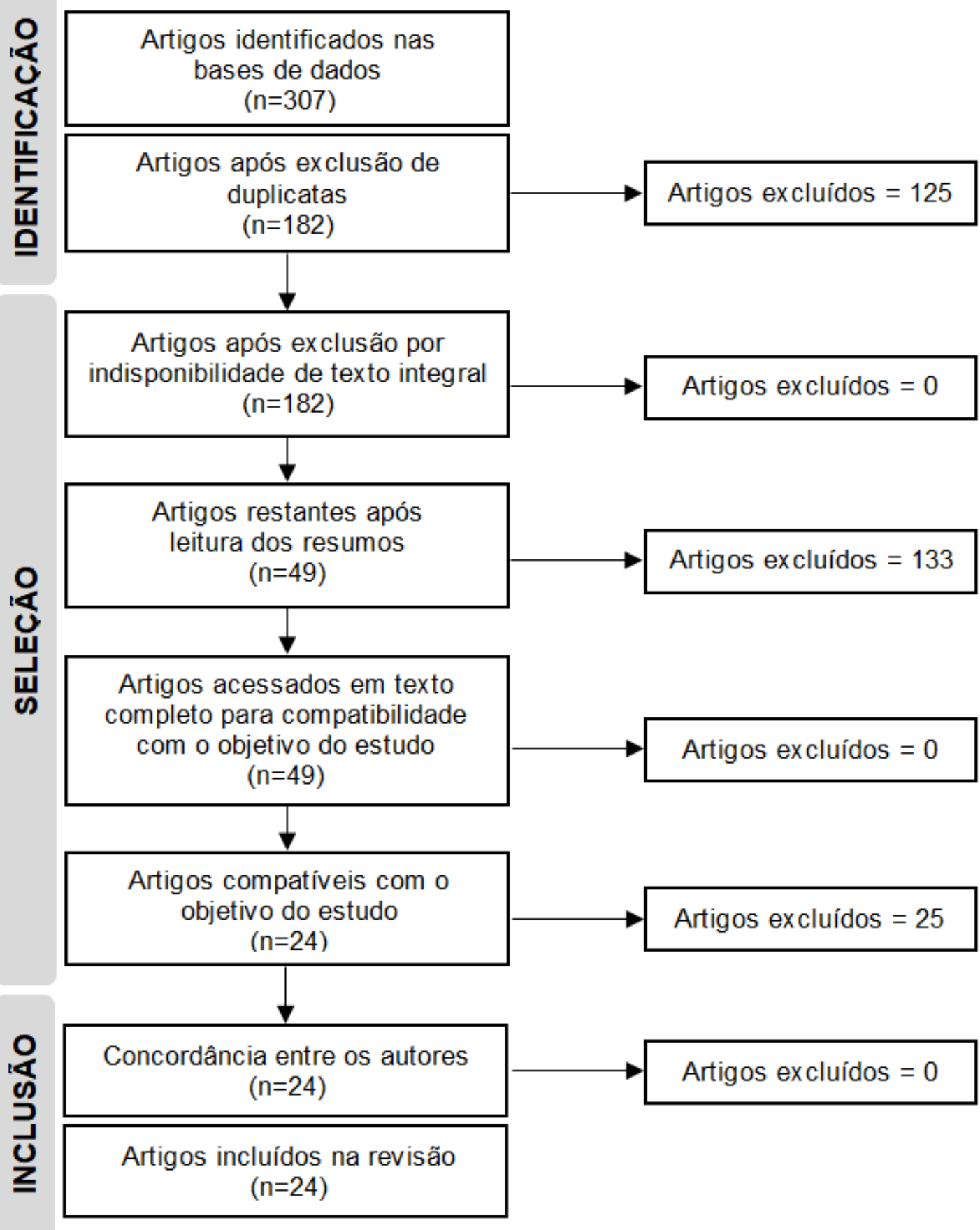

Fonte: Elaborado pelos autores (2021)

Conforme ilustrado no fluxograma, foram encontrados 307 artigos somando os resultados nas duas bases de dados, dos quais 125 foram descartados por duplicidade, restando 182 artigos. Na próxima etapa, realizouse a leitura dos resumos para verificar se estavam compatíveis com o objeto de estudo desta pesquisa. Foram considerados incompatíveis os artigos que: 1) não 
abordavam explicitamente compartilhamento do conhecimento; 2) não abordavam explicitamente situações de crise; e 3) abordavam "crise" na perspectiva voltada ao marketing (como crises de confiança na marca ou produto). Nesta fase foram excluídos 133 artigos, restando 49 artigos.

Por fim, foi realizada a leitura completa dos artigos e verificou-se a disponibilidade na íntegra dos mesmos, sendo que nesta última etapa foram descartados mais 25 artigos. Assim, foram selecionados 24 artigos para 0 procedimento de codificação e análise. O Quadro 1 apresenta a distribuição quantitativa e cronológica dos artigos que compõem a seleção final.

\section{Quadro 1 - Publicações por ano}

\begin{tabular}{|c|c|}
\hline ANO & QUANTIDADE DE ARTIGOS \\
\hline 2017 & 2 \\
\hline 2018 & 3 \\
\hline 2019 & 2 \\
\hline 2020 & 9 \\
\hline 2021 até maio & 8 \\
\hline
\end{tabular}

Fonte: Elaborado pelos autores (2021)

A seleção de 24 artigos compôs a amostra definitiva, que foi novamente lida na íntegra pelos autores, com o objetivo de análise das informações e extração dos principais temas constantes em cada pesquisa individual. Os dados foram categorizados e empregados na integração e discussão final, com finalidade de responder ao objetivo desta revisão sistemática de literatura.

\section{RESULTADOS}

Com o propósito de identificar as principais características de compartilhamento do conhecimento dos últimos quatro anos em situações de crises, o Quadro 2 descreve e sintetiza os artigos selecionados, indicando os autores, ano de publicação, título do artigo e um resumo com as principais constatações derivadas da pesquisa apresentada. 


\section{Quadro 2 - Caracterização dos estudos}

\begin{tabular}{|c|c|}
\hline AUTOR/TíTULO & RESUMO E CONSTATAÇÕES \\
\hline $\begin{array}{l}\text { Hardt (2017) } \\
\text { Who matters for memory: } \\
\text { Sources of institutional memory } \\
\text { in international organization } \\
\text { crisis management. }\end{array}$ & $\begin{array}{l}\text { O estudo identifica quais fatores afetam o } \\
\text { compartilhamento do conhecimento das elites das } \\
\text { Organizações Internacionais ligadas à OTAN, e se } \\
\text { contribuem ou não com o conhecimento para a memória } \\
\text { institucional no gerenciamento de crises. Com } 120 \\
\text { instituições pesquisadas o estudo verificou que existe um } \\
\text { ceticismo das organizações para acreditar na disposição } \\
\text { do governo dos EUA em compartilhar informações } \\
\text { confiáveis e imparciais, pois existe uma crença de que os } \\
\text { EUA repassam essas informações para fins políticos. } \\
\text { Devido à falta de confiança na informação, tem-se uma } \\
\text { menor probabilidade de compartilhá-la, bem como registrá- } \\
\text { la na memória institucional de cada organização. }\end{array}$ \\
\hline $\begin{array}{l}\text { Hoffmann et al. (2017) } \\
\text { United we stand, divided we fall? } \\
\text { Clustered firms' relationships } \\
\text { after the } 2008 \text { crisis. }\end{array}$ & $\begin{array}{l}\text { Foram analisados, de forma qualitativa, distritos industriais } \\
\text { de cerâmica espanhola durante a crise de } 2008 \text { para } \\
\text { identificar a cooperação entre os mesmos. Verificou-se } \\
\text { que o compartilhamento de conhecimento em situações de } \\
\text { crises fez com que as empresas apresentassem menos } \\
\text { cooperação com empresas do mesmo setor, pois eram } \\
\text { consideradas concorrentes. A maior inovação e } \\
\text { compartilhamento do conhecimento ocorreu diretamente } \\
\text { com os fornecedores, pois, devido à crise, as empresas } \\
\text { terceirizaram mais suas atividades, inclusive a P\&D, ou } \\
\text { seja, uma transferência de conhecimento vertical. }\end{array}$ \\
\hline $\begin{array}{l}\text { Utunen et al. (2018) } \\
\text { Knowledge transfer for Ebola } \\
\text { outbreak-production and use of } \\
\text { OpenWHO.org online learning } \\
\text { resources. }\end{array}$ & $\begin{array}{l}\text { Estudou a criação de uma plataforma da OMS, a qual tem } \\
\text { como objetivo compartilhar conhecimento de forma rápida } \\
\text { e confiável para o combate e prevenção de epidemias, } \\
\text { como a do vírus Ebola. Assim, treinamentos estavam } \\
\text { disponíveis um dia após o surto do Ebola em } 2018 \text { em } \\
\text { vários idiomas, fazendo com que países atingidos } \\
\text { conseguissem utilizar desse conhecimento para conter a } \\
\text { epidemia de forma mais efetiva. }\end{array}$ \\
\hline $\begin{array}{l}\text { Eray (2018) } \\
\text { Storytelling in } \\
\text { Communication. }\end{array}$ & $\begin{array}{l}\text { Verificou que contar histórias pode ser uma técnica de } \\
\text { comunicação eficaz no combate de crises organizacionais. } \\
\text { Uma das constatações é que, no mundo digital, as } \\
\text { narrativas podem ser contadas por meio das mídias } \\
\text { sociais, atingindo mais pessoas. Quando a técnica é } \\
\text { corretamente utilizada gera uma proteção da reputação, } \\
\text { ganho de confiança do público e de seus funcionários e, } \\
\text { possivelmente, as instituições passarão por uma crise } \\
\text { acumulando menores danos. }\end{array}$ \\
\hline
\end{tabular}


Hurtado et al. (2018)

Evaluating the frequency of operational research conducted during the 2014-2016 West Africa Ebola epidemic.

Tsouri (2019)

Knowledge transfer in time of crisis: evidence from the Trentino region.
Foi realizada uma análise quantitativa da literatura científica da epidemia de Ebola na África Ocidental, ocorrida em 2014-2016. Verificou que das 3681 publicações encontradas sobre o Ebola apenas 109 eram de pesquisa operacional e, entre elas, apenas 64 foram publicadas depois que a OMS declarou o surto desse vírus. O estudo constatou que houve uma escassez nas pesquisas operacionais que auxiliassem os gestores a formar políticas públicas sólidas e eficazes para o combate de surtos e epidemias.

O estudo avaliou o impacto da diferença entre as redes de colaboração e compartilhamento do conhecimento antes e durante a crise financeira de 2008 em empresas italianas. As principais conclusões foram que as pessoas nas organizações tendem a preferir colaborar apenas com agentes confiáveis e com quem já cooperaram no passado em épocas de crises. Quando não há crises os agentes tendem a expandir a rede de conhecimento da região, realizando novas colaborações com agentes mais distantes e a nível global.

O estudo abordou seis companhias varejistas internacionais que tentaram expandir seus negócios durante a crise financeira asiática de 1997, a recessão norte-americana de 2007 e a crise da dívida soberana

Sung e Goebel (2019)

International Expansion of Retailers: The Role of Technical Expertise, Alliances, and Allocation of Resources in Economic Crises.

Wolor et al. (2020)

The Effectiveness of Virtual Work to Keep Achieving Optimal Performance Amid the Covid-19 Virus Outbreak.

Chellamuthu e Muthu (2020)

Pandemic response protocol of a non-frontline specialty in a multispecialty tertiary health care centre-a pilot model in orthopaedics europeia de 2009, verificando três tipos de transferência de conhecimento que potencializavam o sucesso: (1) desenvolvimento a longo prazo de conhecimento técnico além do mercado doméstico; (2) alianças com parceiros locais no mercado alvo, com os quais a empresa pode se engajar na transferência de conhecimento entre blocos culturais, e (3) alocação focalizada de recursos da empresa para a transferência de conhecimento.

O estudo analisou sistematicamente abordagens para melhorar a implementação de trabalho virtual durante a pandemia da COVID-19. Verificou que, para uma melhor eficácia, deve haver a promoção do compartilhamento do conhecimento, para isso o artigo sugere: apoio gerencial; infraestrutura compatível com o trabalho; nova política e novas regras; confiança, comunicação e feedback entre os membros; aplicação da tecnologia adequada.

Criou-se um protocolo de resposta pandêmica (PREP) com o objetivo de elaborar uma resposta mínima essencial para uma especialidade médica, neste caso a ortopedia. Assim, foi possível reduzir os efeitos causados pela COVID-19 em um centro de saúde terciário multiespecializado. O artigo mostra a importância do compartilhamento das melhores práticas envolvendo várias organizações e colaborações. 
Mazuze et al. (2020)

Exploring forgotten knowledge to enhance effective knowledge deliver in aid organizations.

Bakos (2020)

Human-machine communication and crisis management: an Automotive industry case study.

Lee et al. (2020)

\section{Enhancing}

knowledge sharing through diversity-oriented leadership and strategic internal communication during the COVID-19 outbreak.

Cargnel et al. (2020)

Improving laboratory diagnostic capacities of emerging diseases using knowledge mapping.
O artigo inclui a exploração dos três tipos de conhecimento no setor humanitário em época da pandemia ocasionada pela COVID-19 - o conhecimento tácito e o explícito. Com a finalidade de melhorar o compartilhamento do conhecimento nas organizações em épocas de crise recomendou: remodelar a força de trabalho contingente; adaptar a gestão da mobilidade dos funcionários; realizar abordagens inovadoras de coaching; desregulamentar 0 uso da tecnologia para a transferência eficiente de conhecimento entre as pessoas.

O estudo analisa a gestão de crises na indústria de abastecimento automotivo, em particular, como se dá a comunicação entre homem e máquina. Verificou que nessas indústrias (exceto nas áreas de segurança ocupacional) não existem procedimentos gerais para situações imprevisíveis, fazendo com que as decisões sejam tomadas em nível de presidência, o que promove uma gestão de crises com decisões demoradas e burocráticas.

Segundo o estudo, uma liderança orientada à diversidade contribui para uma comunicação interna transparente durante uma crise e aumenta a satisfação dos funcionários com relação às necessidades de autonomia, competência e relacionamento. Além disso, a comunicação interna promove a satisfação das necessidades intrínsecas dos funcionários, tornando-os mais engajados no trabalho e compartilhando seus conhecimentos com mais frequência durante a crise.

Um estudo na Bélgica sobre diagnóstico de doenças animais emergentes e reemergentes identificou a necessidade do mapeamento do conhecimento nessas instituições em níveis nacionais e internacionais. Com esse mapeamento a preparação e a capacidade de respostas do país frente a esses desafios se torna mais rápida e eficaz.
Bustamante et al. (2020)

The Haiti Humanitarian Response Course: A Novel Approach to Local Responder Training in International Humanitarian Response
Os países mais afetados por desastres naturais são os que possuem uma capacidade limitada de resposta para essas crises. No Haiti, em 2017, foi criado o Curso: "Resposta Humanitária do Haiti". Com esse treinamento, desenvolvido por meio de parcerias internacionais, foi possível criar um modelo potencial para o treinamento humanitário e de desastres, tanto para o Haiti como para outros países. Essa capacitação tornou possível melhorar a utilização de recursos, de compartilhamento do conhecimento e de resposta a desastres. 
Urick (2020)

Generational Differences and COVID-19: Positive Interactions in Virtual Workplaces.

Durante a pandemia da COVID-19 muitas empresas no mundo fizeram com que os trabalhadores mudassem para trabalho online. Essa mudança repentina para o trabalho remoto aumentou as percepções negativas que os indivíduos podem ter de colegas de outras gerações, levando a uma quebra nas interações e afetando o compartilhamento do conhecimento.

Em grandes crises, como a evacuação da cidade de Dunquerque ou a gestão da pandemia da COVID-19 realizada por Taiwan, foi constatada a importância das colaborações público-privadas que promovessem um compartilhamento do conhecimento em seu núcleo para a solução de momentos de crises. Para uma efetiva colaboração identificou-se a necessidade das seguintes características: 1) empreendedorismo entre equipes; 2) forte motivações dos participantes; 3) liderança governamental com expertise adequada e uma linha de comando unitária; 4) ativação do conhecimento dependente das circunstâncias; 5) compartilhamento do conhecimento voltado a resolução dos problemas; e 6) colaboração como um processo de aprendizagem inserido na dinâmica do conhecimento.

\section{$\mathrm{Ng}$, Fong e Lo (2021)}

Dynamic knowledge management in response to the pandemic outbreak: an interinstitutional risk-based approach to sustainability
O estudo analisou o caso de Hong Kong para a proteção de saúde pública durante a pandemia COVID-19, na qual a cidade utilizou uma estrutura integrativa e interinstitucional baseada em riscos para otimizar o combate à pandemia. Colaboração e compartilhamento do conhecimento entre instituições públicas e privadas foram necessários para a implementação de políticas de saúde pública e medidas de controle de maneira responsiva.
Tavra, Racetin e Peros (2021)

The role of crowdsourcing and social media in crisis mapping: a case study of a wildfire reaching Croatian City of Split.

Montani e Stagliano (2021)

Innovation in times of pandemic: The moderating effect of knowledge sharing on the relationship between COVID-19induced job stress and employee innovation.
O artigo analisa a importância do uso de dados georreferenciados de mídia social no combate a incêndios de uma cidade da Croácia. Constatou que a utilização de múltiplas fontes de informação impacta positivamente o compartilhamento do conhecimento, consequentemente, há uma melhora na gestão de desastres. Assim, recomenda-se que a utilização de processos de crowdsourcing podem ser incorporados ao gerenciamento de desastres.

Em um estudo com 61 funcionários de empresas britânicas de P\&D examinou o efeito do compartilhamento do conhecimento na inovação em ambientes de estresse gerado pela pandemia da COVID-19. Verificou que o compartilhamento do conhecimento moderou o estresse causado pela pandemia e promoveu a inovação dos funcionários. 


\begin{tabular}{|c|c|}
\hline $\begin{array}{ll}\text { Co-Operative } & \text { Learning and } \\
\text { Resilience to } & \text { COVID-19 in a } \\
\text { Small-Sized South African } \\
\text { Enterprise. }\end{array}$ & $\begin{array}{l}\text { A pesquisa verificou, por meio de um estudo de caso em } \\
\text { uma pequena organização da África do Sul, que utilizar } \\
\text { uma estrutura baseada em compartilhamento do } \\
\text { conhecimento, mediante o aprendizado cooperativo, induz } \\
\text { um maior desempenho inovador e estruturas mais } \\
\text { eficientes de utilização de recursos durante as crises. Com } \\
\text { o compartilhamento do conhecimento adequado a } \\
\text { organização tende a ter uma maior capacidade para resistir } \\
\text { às crises e manter sua sustentabilidade após este período. }\end{array}$ \\
\hline $\begin{array}{l}\text { Sakusic et al. (2021) } \\
\text { Rapid, multimodal, critical care } \\
\text { knowledge-sharing platform for } \\
\text { COVID-19 pandemics. }\end{array}$ & $\begin{array}{l}\text { Para abordar efetivamente o excesso de informações } \\
\text { novas e não filtradas, com o apoio da Organização Mundial } \\
\text { de Saúde, foi criada uma plataforma de intervenção } \\
\text { multimodal de tele-educação em instituições de cuidados } \\
\text { a pacientes de COVID-19. Utilizando plataformas de } \\
\text { mídias sociais foi observado um alto nível de interação com } \\
\text { os mais de } 2000 \text { participantes, mostrando que a tele- } \\
\text { educação é uma forma viável e eficaz, de baixo custo e de } \\
\text { alta interação entre os participantes, resultando em um } \\
\text { efetivo compartilhamento do conhecimento. }\end{array}$ \\
\hline $\begin{array}{l}\text { Alonazi (2021) } \\
\text { Building learning organizational } \\
\text { culture during COVID-19 } \\
\text { outbreak: a national study. }\end{array}$ & $\begin{array}{l}\text { Na crise ocasionada pela COVID-19, um estudo com } 1500 \\
\text { trabalhadores de instituições de saúde na Arábia Saudita } \\
\text { constatou que é necessária a implementação de várias } \\
\text { dimensões de aprendizagem organizacional, } \\
\text { principalmente as extrínsecas, tais como as conexões } \\
\text { externas e as colaborações. }\end{array}$ \\
\hline $\begin{array}{l}\text { Tseng, Bui e Lim (2021) } \\
\text { Resource utilization model for } \\
\text { sustainable solid waste } \\
\text { management in Vietnam: A crisis } \\
\text { response hierarchical structure. }\end{array}$ & $\begin{array}{l}\text { O gerenciamento de resíduos sólidos nas cidades do } \\
\text { Vietnã em época de COVID-19 criou obstáculos e } \\
\text { incertezas. Dessa forma, houve a necessidade de uma } \\
\text { colaboração entre os municípios. Alguns critérios foram } \\
\text { essenciais para a efetividade do equilíbrio da gestão: 1) a } \\
\text { estrutura de política e regulamentação; } 2 \text { ) } \\
\text { compartilhamento de informações e conhecimentos; e 3) } \\
\text { intervenção ocupacional, bons padrões de emprego e } \\
\text { apoio aos parâmetros da cidade. }\end{array}$ \\
\hline $\begin{array}{l}\text { Christaa e Kristinae (2021) } \\
\text { The effect of product innovation } \\
\text { on business performance during } \\
\text { COVID } 19 \text { pandemic. }\end{array}$ & $\begin{array}{l}\text { Os autores aplicaram um modelo conceitual, baseado em } \\
\text { compartilhamento do conhecimento e inovação, para } \\
\text { aumentar o volume de negócios em duas províncias na } \\
\text { Indonésia afetadas pela pandemia da COVID-19. O estudo } \\
\text { sugere que é necessário encorajar os agentes } \\
\text { empresariais de forma proativa a compartilhar o } \\
\text { conhecimento com base na orientação do cliente, na } \\
\text { disseminação das melhores práticas dos concorrentes e } \\
\text { na promoção de uma interface de coordenação para } \\
\text { melhoria do desempenho comercial. }\end{array}$ \\
\hline
\end{tabular}

Fonte: Elaborado pelos autores (2021) 
De acordo com as informações do Quadro 2, observou-se uma grande heterogeneidade dos estudos quanto às características de métodos de pesquisa, tamanho das amostras, localidade e também diversidade de periódicos. Verificou-se que muitas publicações ocorreram nos anos de 2020 e 2021, e isso se deve ao fato das publicações serem referentes à pandemia ocasionada pela COVID-19. Dos 24 artigos estudados, 13 trataram sobre compartilhamento do conhecimento durante a pandemia da COVID-19.

Os principais assuntos identificados na literatura estudada sobre compartilhamento do conhecimento foram os seguintes: confiança, memória institucional, diversidade nas organizações, efeitos da crise no compartilhamento do conhecimento, ferramentas para compartilhamento do conhecimento, técnicas e estratégias para o compartilhamento do conhecimento, e barreiras para o compartilhamento do conhecimento. O Quadro 3 apresenta os temas emergentes nas pesquisas, indicando os autores e o ano de publicação.

\section{Quadro 3 - Principais temas presentes nos artigos analisados}

\begin{tabular}{|c|c|c|}
\hline TEMA & CONTEÚDO & AUTORES \\
\hline Confiança & $\begin{array}{l}\text { Importância dos laços de confiança } \\
\text { mútua. }\end{array}$ & $\begin{array}{r}\text { Hardt (2017) } \\
\text { Habiyaremye (2021) }\end{array}$ \\
\hline $\begin{array}{l}\text { Memória } \\
\text { Institucional }\end{array}$ & $\begin{array}{l}\text { Mostra a importância da memória } \\
\text { institucional. }\end{array}$ & Hardt (2017) \\
\hline $\begin{array}{l}\text { Diversidade nas } \\
\text { Organizações }\end{array}$ & $\begin{array}{l}\text { Presença da diversidade (gênero, } \\
\text { idade cor, etc.), seus efeitos no } \\
\text { compartilhamento do conhecimento } \\
\text { em épocas de crises e a importância } \\
\text { de uma liderança orientada à } \\
\text { diversidade. }\end{array}$ & $\begin{array}{r}\text { Hardt (2017) } \\
\text { Lee et al. (2020) } \\
\text { Urick (2020) }\end{array}$ \\
\hline $\begin{array}{l}\text { Efeitos da Crise no } \\
\text { Compartilhamento } \\
\text { do Conhecimento }\end{array}$ & $\begin{array}{l}\text { Quais são os efeitos, as vantagens e } \\
\text { desvantagens de promover o } \\
\text { comportamento voltado ao } \\
\text { compartilhamento do conhecimento e } \\
\text { à inovação em épocas de crises. }\end{array}$ & $\begin{array}{r}\text { Hoffmann et al. (2017) } \\
\text { Tsouri (2019) } \\
\text { Montani e Stagliano (2021) }\end{array}$ \\
\hline $\begin{array}{l}\text { Ferramentas para } \\
\text { Compartilhamento } \\
\text { do Conhecimento }\end{array}$ & $\begin{array}{l}\text { Plataformas online, sistemas } \\
\text { colaborativos, sistemas integrados e } \\
\text { mapeamento do conhecimento } \\
\text { melhoram os resultados esperados no } \\
\text { compartilhamento do conhecimento } \\
\text { em épocas de crises. }\end{array}$ & $\begin{array}{r}\text { Utunen et al. (2018) } \\
\text { Bustamante et al. (2020) } \\
\text { Cargnel et al. (2020) } \\
\text { Tavra, Racetin e Peros (2021) } \\
\text { Sakusic et al. (2021) } \\
\text { Alonazi (2021) }\end{array}$ \\
\hline
\end{tabular}




\begin{tabular}{|c|c|c|}
\hline $\begin{array}{l}\text { Técnicas e } \\
\text { Estratégias para o } \\
\text { Compartilhamento } \\
\text { do Conhecimento }\end{array}$ & $\begin{array}{l}\text { Diversas técnicas e estratégias de } \\
\text { compartilhamento do conhecimento } \\
\text { são apresentadas para que as } \\
\text { organizações superem momentos de } \\
\text { crises ou desastres. }\end{array}$ & $\begin{array}{r}\text { Eray (2018) } \\
\text { Sung e Goebel (2019) } \\
\text { Chellamuthu e Muthu (2020) } \\
\text { Wolor, Aminah e Martono (2020) } \\
\text { Mazuze et al. (2020) } \\
\text { Lee et al. (2020) } \\
\text { Huang e Shih-Wei (202) } \\
\text { Ng, Fong e Lo (2021) } \\
\text { Habiyaremye (2021) } \\
\text { Tseng, Bui e Lim (2021) } \\
\text { Christaa e Kristinae (2021) }\end{array}$ \\
\hline $\begin{array}{l}\text { Barreiras para o } \\
\text { Compartilhamento } \\
\text { do Conhecimento }\end{array}$ & $\begin{array}{l}\text { Algumas organizações ou grupos } \\
\text { tendem a ter mais dificuldade para } \\
\text { superar situações imprevistas e de } \\
\text { crises, devido a algumas barreiras no } \\
\text { compartilhamento do conhecimento. }\end{array}$ & $\begin{array}{r}\text { Hurtado et al. (2018) } \\
\text { Bakos (2020) }\end{array}$ \\
\hline
\end{tabular}

Fonte: Elaborado pelos autores (2021)

Em relação a Confiança, Hardt (2017) verificou que as elites da OTAN são mais propícias a compartilhar o conhecimento entre elas quando recebem de uma fonte confiável. Neste estudo de caso, o autor concluiu que quando recebiam informações do governo dos EUA havia muito ceticismo por parte das elites, devido aos erros da inteligência americana que provocaram a guerra no Iraque em 2003. De acordo com o autor, mesmo após 10 anos deste fato, a reputação na qualidade das informações do governo dos EUA ainda estava sendo julgada pelos recebedores, e havia medo dessa informação conter intenções políticas.

Habiyaremye (2021), no seu estudo constatou que é necessário procurar fortalecer os laços de confiança mútua entre os membros das colaborações, para que seja possível unir os pontos fortes de cada um e, assim, enfrentar as crises com melhores resultados para todas as organizações. Dessa forma, a confiança dentro e fora das organizações em situações de crises aumenta a chance de sucesso. (HARDT, 2017; HABIYAREMYE, 2021).

O segundo tópico identificado nos estudos refere-se à Memória Institucional. No estudo de caso das elites da OTAN percebeu-se que, em determinadas situações de crises, ao invés dos agentes consultarem os bancos de dados da instituição, preferem procurar conhecimentos diretamente com as pessoas ou em anotações privadas (HARDT, 2017). Mesmo quando os documentos estão dispersos na organização, ainda assim, verificou-se a 
importância de os conhecimentos estarem disponíveis em formas explícitas (HARDT, 2017).

A Diversidade nas Organizações consiste no quanto uma organização respeita e promove a diversidade de seus trabalhadores, tais como: gênero, faixa etária, opção sexual, cor da pele, etc. Um estilo de liderança orientado à diversidade na organização promove uma comunicação transparente $\mathrm{e}$ satisfação de necessidades intrínsecas dos funcionários, o que aumenta o compartilhamento do conhecimento e o engajamento no trabalho durante as crises (LEE et al., 2021).

Segundo Hardt (2017), a diferença etária nas instituições foi geralmente considerada benéfica, pois os colaboradores mais velhos da OTAN apresentaram maior propensão a compartilhar o conhecimento - seja verbalmente ou por registro - do que as elites mais jovens. É importante a promoção de compartilhamento do conhecimento mútuo entre membros de várias faixas etárias pois, além de criar um melhor clima organizacional, os conhecimentos se complementam e oferecem melhor preparação para o enfrentamento de situações de crises (HARDT, 2017; URICK, 2020).

Durante a pandemia da COVID-19 muitas organizações foram obrigadas a mudar seus ambientes físicos para o online (URICK, 2020). Esta mudança acentuou as percepções negativas das diferenças geracionais em relação à tecnologia, reduzindo as interações entre os mais jovens e os mais velhos, causando uma perda importante no compartilhamento do conhecimento e em resultados mais efetivos na organização (URICK, 2020). Sendo assim, é importante que os gestores entendam a importância das percepções geracionais, pois há impacto na forma de trabalhos em conjunto entre diferentes idades; caso estas interações sejam positivas, existe maior chance de a organização ser bem-sucedida em épocas de crises (LEE et al., 2020; URICK, 2020).

O quarto tópico encontrado é Efeitos da Crise no Compartilhamento do Conhecimento. De acordo com Hoffmann et al. (2017), em momentos de crises há uma redução de confiança entre os parceiros horizontais: as organizações desconfiam que, devido à maior competição entre as empresas, haverá uma 
maior imitação ou um comportamento oportunista da concorrência. Em contrapartida, devido à necessidade de redução de custos, as empresas diminuem seu escopo e se especializam. Assim, ocorre uma maior terceirização de diversas atividades aos fornecedores (como P\&D e marketing), o que aumenta a interdependência organizacional e, consequentemente, eleva 0 compartilhamento do conhecimento vertical (HOFFMANN et al., 2017).

Outro estudo identificou que, em períodos de crises, as pessoas preferem realizar colaborações com sujeitos que já realizaram no passado, ao invés de procurar novos parceiros, deixando a rede de conhecimento inerte (TSOURI, 2019). Já em períodos de baixos riscos há uma tendência de expansão na rede de conhecimento das organizações, as quais buscam colaborações e conhecimentos de novos parceiros, e de regiões mais distantes (TSOURI, 2019).

Segundo Tsouri (2019), durante os períodos de crises, mesmo sujeitos próximos e que compartilham características em comum são evitados nas colaborações. O compartilhamento do conhecimento tende a ocorrer com atores com quem já foram realizadas cooperações repetidamente no passado, criando uma confiança interorganizacional ainda maior entre os agentes (TSOURI, 2019).

Durante o período da pandemia um estudo em empresas de P\&D britânicas constatou que, quando o compartilhamento do conhecimento foi maior, os trabalhadores tiveram uma redução no estresse e um aumento na promoção do comportamento inovador (MONTANI; STAGLIANO, 2021). Neste estudo verificou-se que, mesmo com o estresse causado pela pandemia da COVID-19, o compartilhamento do conhecimento entre os funcionários estava elevado, e um aumento no desempenho inovador na organização foi observado (MONTANI; STAGLIANO, 2021).

O tópico Ferramentas para Compartilhamento do Conhecimento está presente em seis artigos ( $25 \%$ da seleção analisada). Constatou-se que as plataformas online e o crowdsourcing são ferramentas muito utilizadas em situações de desastres ou de crises (UTUNEN et al., 2018; BUSTAMANTE et al., 2020; TAVRA; RACETIN; PEROS; 2021; SAKUSIC, 2021).

Em situações de surtos, pandemias ou desastres muitas organizações 
contam com o suporte de plataformas de aprendizagem online, com o apoio de organismos como a Organização Mundial de Saúde ou Organizações das Nações Unidas, como é o caso das seguintes plataformas: OpenWho.org (UTUNEN et al., 2018); tele-educação com plataformas de mídias sociais (SAKUSIC, 2021); treinamentos em várias línguas com vários parceiros internacionais para situações de desastres locais (BUSTAMANTE et al., 2020). $O$ baixo custo e a velocidade de plataformas online trazem benefícios importantes e uma utilização melhor dos recursos no combate a diversas crises ao redor do mundo (UTUNEN et al., 2018; BUSTAMANTE et al., 2020; SAKUSIC, 2021).

Segundo Tavra, Racetin e Peros (2021), as mídias sociais têm desempenhado um importante papel em situações de crises locais provocadas por desastres, devido à velocidade no trânsito da informação. Prefeituras que adotam múltiplas fontes de compartilhamento do conhecimento (em mídias sociais, sites, além do uso de informações geoespaciais) têm apresentado melhores resultados no gerenciamento dessas crises do que aquelas que só utilizam os sistemas de comunicação tradicionais (TAVRA; RACETIN; PEROS; 2021).

Outras ferramentas, quando já estabelecidas antes das crises, auxiliam as organizações a superar estes momentos de forma mais efetiva (ALONAZI, 2021; CARGNEL et al., 2020) Por exemplo, sistemas integrados de conhecimento, quando são bem utilizados, criam uma cultura de compartilhamento do conhecimento que ajuda na construção de relacionamentos eficazes e servem como um ambiente de aprendizagem contínua, promovendo melhores resultados durante as crises (ALONAZI, 2021). Outro estudo revelou a utilidade do mapeamento do conhecimento para ter uma resposta mais rápida e eficaz em situações de crises e pandemias, principalmente quando utilizado para identificar os conhecimentos de organizações mais distantes, que se encontram até mesmo em outros países (CARGNEL et al., 2020).

O tópico presente no maior número de artigos ( $45 \%$ do total) refere-se às Técnicas e Estratégias para o Compartilhamento do Conhecimento. 
Diversas técnicas e estratégias de compartilhamento do conhecimento em situações de crises foram estudadas para derivar e sintetizar as melhores práticas.

Segundo Eray (2018) utilizar o storytelling pode diminuir o estresse e os sentimentos negativos durante as crises. As histórias e as narrações têm o poder de moldar a cultura do ambiente e a imagem da organização com muito mais força na atualidade, e as mídias sociais podem influenciar milhões de pessoas (ERAY, 2018). Ainda segundo o autor, quando utilizadas de forma eficaz, as histórias contadas por meio das mídias sociais protegem a reputação, aumentam a confiança do público e melhoram o desempenho organizacional em períodos de crises.

Já para os autores Lee et al. (2020), em momentos de crises organizacionais, faz-se necessário amenizar os níveis de ambiguidade e incerteza. Por isso, as organizações devem incentivar uma comunicação transparente e participativa entre os funcionários da instituição (LEE et al., 2020; TSENG, BUI, LIM, 2021).

Em situações de desastres e pandêmicas é de grande valia criar Protocolos de Respostas para um melhor gerenciamento da crise (CHELLAMUTHU; MUTHU, 2020). Outras formas observadas são colaborações público-privadas para o enfrentamento desses eventos (HUANG; SHIH-WEI, 2020; NG; FONG; LO, 2021). Segundo Huang e Shih-Wei (2020) existem certas condições para uma colaboração bem-sucedida em situações de crise: fortes motivações dos participantes; liderança do governo com especialização adequada; empreendedorismo entre as equipes formadas; linha de comando unitária.

O último tópico encontrado nos artigos é Barreiras para o Compartilhamento do Conhecimento Durante a Crise. Bakos (2020) verificou que, em indústrias altamente regulamentadas e padronizadas, quando ocorrem situações imprevistas ou de crises, os funcionários apresentam um comportamento ambíguo, deixando de tomar decisões em situações determinadas. Devido à falta de disposição para situações imprevistas e à ausência de autonomia dos funcionários, as decisões a serem tomadas são 
direcionadas aos CEOs ou à diretoria, prejudicando a agilidade das organizações devido à lentidão nas decisões (BAKOS, 2020).

\section{CONCLUSÕES}

Esta revisão da literatura evidenciou em tópicos e categorias específicos as principais pesquisas referentes ao compartilhamento do conhecimento em situações de crises e, numa visão mais ampla, abordou também a gestão do conhecimento. Desta forma, constata-se que o objetivo proposto para este estudo foi alcançado. A partir do método PRISMA as publicações foram selecionadas e, posteriormente, categorizadas e analisadas, definindo os principais tópicos utilizados pelos autores nas pesquisas, os quais foram identificados como: i) confiança; ii) memória institucional; iii) diversidade nas organizações; iv) efeitos da crise no compartilhamento do conhecimento; v) ferramentas para compartilhamento do conhecimento; vi) técnicas e estratégias para o compartilhamento do conhecimento; e vii) barreiras para o compartilhamento do conhecimento.

Verificou-se também que, com o início da pandemia da COVID-19 em 2020, o número de artigos publicados sobre este tema aumentou significativamente, representando mais de $50 \%$ dos artigos publicados, e a representação majoritária também foi constatada nesta revisão. Apesar do aumento de publicações, ainda existem lacunas a serem preenchidas, principalmente em estudos que sejam capazes de analisar as estratégias emergentes e mais eficazes de compartilhamento do conhecimento organizacional.

O presente estudo limitou-se a conter uma discussão de pesquisas relacionadas ao compartilhamento do conhecimento em situações de crises com publicações do período de janeiro de 2017 até maio de 2021. Como este estudo utilizou pesquisas com termos em inglês, encontrou apenas artigos publicados neste idioma. A presente estratégia, apesar de não conter artigos em português ou outras línguas, proporcionou uma amostra imparcial dos estudos relevantes sobre o tema no idioma predominante na produção acadêmica internacional.

Assim, devido à escassez de pesquisas sobre estratégias mais amplas 
para o compartilhamento do conhecimento em épocas de crises, sugere-se que estudos futuros identifiquem as principais práticas de compartilhamento do conhecimento para maximizar os resultados organizacionais em situações de crises. Estudos futuros também poderiam explorar o compartilhamento do conhecimento em crises contendo um período temporal maior, para que possibilite, o entendimento sobre a evolução das pesquisas ao longo dos anos.

\section{REFERÊNCIAS}

AHMAD, F.; KARIM, M. Impacts of knowledge sharing: a review and directions for future research. Journal of Workplace Learning, v. 31, n. 3, p. 207-230, 2019.

ALI, A. A.; PARIS, L.; GUNASEKARAN, A. Key factors influencing knowledge sharing practices and its relationship with organizational performance within the oil and gas industry. Journal of Knowledge Management, v. 23, n. 9, p. 18061837, 2019.

ALONAZI, W. B. Building learning organizational culture during COVID-19 outbreak: a national study. BMC Health Services Research, v. 21, 2021.

ALVARENGA NETO, R. C. D. Gestão do conhecimento em organizações: proposta de mapeamento conceitual integrativo. São Paulo: Saraiva, 2008.

ASRAR-UL-HAQ, M.; ANWAR, S. A systematic review of knowledge management and knowledge sharing: Trends, issues, and challenges. Cogent Business \& Management, v. 3, n. 1, p. 1-17, 2016.

BAKOS, L. Human-machine communication and crisis management: an Automotive industry case study. Sciendo, v. 12, n. 1, p. 23-27, 2020.

BANSAL, P.; KIM, A.; WOOD, M. O. Hidden in plain sight: The importance of scale in organizations 'attention to issues. Academy of Management Review, v. 43, n. 2, p. 217-241, 2018.

BUSTAMANTE, N. D.; ROUHANI, S. A.; KIVLEHAN, S.; CHECKETT, K. A.; ISRAEL, K.; TOUSSAINT, S.; KAYDEN, S. The Haiti Humanitarian Response Course: A Novel Approach to Local Responder Training in International Humanitarian Response. Prehospital and Disaster Medicine, v. 35, n. 2, p. 220-224, 2020.

ÇAKAR, K. Critical success factors for tourist destination governance in times of crisis: a case study of Antalya, Turkey. Journal of Travel and Tourism Marketing, v. 35, n. 6, p. 786-802, 2018. 
CARGNEL, M.; BIANCHINI, J.; WELBY, S.; KOENEN, F.; VAN DER STEDE, Y.; DE CLERCQ, K.; SAEGERMAN, C. Improving laboratory diagnostic capacities of emerging diseases using knowledge mapping. Transboundary and Emerging Diseases, v. 68, n. 3, p.1175-1189, 2020.

CHELLAMUTHU, G.; MUTHU, S. Pandemic response protocol of a nonfrontline specialty in a multispecialty tertiary health care centre a pilot model in orthopaedics. International Orthopaedics, v. 4, n. 8, p. 1481-1487, 2020.

CHRISTAA, U. R.; KRISTINAE, V. The effect of product innovation on business performance during COVID 19 pandemic. Uncertain Supply Chain Management, v. 9, p. 151-159, 2021.

CRONIN, P.; RYAN, F.; COUGHLAN, M. Undertaking a literature review: a step-by-step approach. British Journal of Nursing, v.17, n. 1, p. 38-43, 2008.

CUMMINGS, J. N. Work groups, structural diversity, and knowledge sharing in a global organization. Management Science, v. 50, n. 3, p. 352-364, 2004.

DAVENPORT, T. H., PRUSAK, L. Conhecimento empresarial. Rio de Janeiro: Campus, 1998.

DIAS, S. E. F.; SADAO, I. E.; VILAS, E. P. B. Effectuation theoretical debate: systematic review and research agenda. Innovation \& Management Review, v. 17 , n. 1 , p. $41-57,2019$.

DICKMANN, P.; BIEDENKOPF, N.; KEEPING, S.; EICKMANN, M.; BECKER, $S$. Risk communication and crisis communication in infectious disease outbreaks in Germany: What is being done, and what needs to be done.

Disaster Medicine and Public Health Preparedness, v. 8, n. 3, p. 206-211, 2014.

ERAY, T. E. Storytelling in Crisis Communication. Online Journal of Communication and Media Technologies, v. 8, n. 2, p. 131-144, 2018.

FAHIMNIA, B.; SARKIS, J.; DAVARZANI, H. Green supply chain management: A review and bibliometric analysis. International Journal of Production Economics, Elsevier, v. 162, p. 101-114, 2015.

FOSS, N. J.; PEDERSEN, T. Transferring knowledge in MNCs: The role of sources of subsidiary knowledge and organizational context. Journal of International Management, v. 8, n. 1, p. 49-67, 2002.

GRANT, R. M. Toward a knowledge-based theory of the firm. Strategic Management Journal, v. 17, p. 109-122, 1996.

GRAY, S.; SINGER, A.; SCHMITT-OLABISI, L.; INTRONE, J.; HENDERSON, $J$. Identifying the Causes, Consequences, and Solutions to the Flint Water Crisis Through Collaborative Modeling. Environmental Justice, v. 10, n. 5, p. 154-161, 2017. 
GROTTO, D. O Compartilhamento do Conhecimento nas Organizações. In: ANGELONI, M. T. (coord.). Organizações do conhecimento: infra-estrutura, pessoas e tecnologia. São Paulo: Saraiva, 2003. p. 185-195.

GRUNNAN, T.; FRIDHEIM, H. Planning and conducting crisis management exercises for decision-making: the do's and don'ts. EURO Journal on Decision Processes, v. 5, n. 1-4, p. 79-95, 2017.

HABIYAREMYE, A. Co-Operative Learning and Resilience to COVID-19 in a Small-Sized South African Enterprise. Sustainability, v. 13, n. 4, p. 1-17, 2021.

HARDT, H. Who matters for memory: Sources of institutional memory in international organization crisis management. The Review of International Organizations, v. 13, n. 3, p. 457-482, 2017.

HOFFMANN, V. E.; BELUSSI, F.; MARTÍNEZ-FERNÁNDEZ, M. T.; REYES JR, E. United we stand, divided we fall? Clustered firms' relationships after the 2008 crisis. Entrepreneurship \& Regional Development, v. 29, n. 7, p. 735-758, 2017.

HUANG, C. Y.; SHIH-WEI, F. Public-private collaborations in a Dunkirk moment. Knowledge Management Research and Practice, (ahead of print), p. 1-6, 2020.

HURTADO, C.; MEYER, D.; SNYDER, M.; NUZZO, J. B. Evaluating the frequency of operational research conducted during the 2014-2016 West Africa Ebola epidemic. International Journal of Infectious Diseases, v. 77, p. 29-33, 2018.

LEE, Y.; TAO, W.; LI, J. Y. Q.; SUN, R. Enhancing employees' knowledge sharing through diversity-oriented leadership and strategic internal communication during the COVID-19 outbreak. Journal of Knowledge Management, v. 25, n. 6, p. 1526-1549, 2020.

MACHADO, H. P. V.; ELIAS, M. L. G. G. R. Gestão do conhecimento: constituição do campo, temas e perspectivas de pesquisas. Transinformação, Campinas, v. 32, 2020.

MAZUZE, J. A.; TURNER, G.; DIABIRA, D.; KONATE, C. Exploring forgotten knowledge to enhance effective knowledge deliver in aid organisations. In: INTERNATIONAL CONFERENCE ON INTELLECTUAL CAPITAL, KNOWLEDGE MANAGEMENT \& ORGANISATIONAL LEARNING, 17., 2020. Proceedings [...] 2020. Disponível em: https://pesquisa.bvsalud.org/globalliterature-on-novel-coronavirus-2019-ncov/resource/pt/covidwho-1000941. Acesso em: 17 abr. 2021.

MISCHIATTI, J. A. W.; MOLETA, E. R.; CATAPAN, A. City Manager: uma análise bibliométrica de 1950 a 2016. Revista Paranaense de

Desenvolvimento, v. 38, p. 181-193, 2017. 
MOHER, D.; LIBERATI, A.; TETZLAFF, J.; ALTMAN, D. G.; PRISMA GROUP. Preferred Reporting Items for Systematic Reviews and Meta-Analyses: The PRISMA Statement. Physical Therapy, v. 89, n. 9, p. 873-880, 2009.

MONTANI, F.; STAGLIANO, R. Innovation in times of pandemic: The moderating effect of knowledge sharing on the relationship between COVID-19induced job stress and employee innovation. R\&D Management, (ahead of print), 2021.

NG, A.; FONG, B.; LO, M. F. Dynamic knowledge management in response to the pandemic outbreak: an interinstitutional risk-based approach to sustainability. Knowledge Management Research \& Practice, p. 1-9, 2021.

PAULIN, D.; SUNESON, K. Knowledge transfer, knowledge sharing and knowledge barriers: Three blurry terms in KM. The Electronic Journal of Knowledge Management, v. 10 n.1, p. 81-91, 2012.

PERSSON, A.; STIRNA, J.; AGGESTAM, L. How to disseminate professional knowledge in healthcare: The case of Skaraborg hospital. Journal of Cases on Information Technology (JCIT), v. 10, n. 4, p. 41-64, 2008.

PERRY, A.; HAMMOND, N. Systematic Reviews: The Experiences of a PhD Student. Psychology Learning and Teaching, v. 2, n. 1, p. 32-35, 2002.

SAKUSIC, A.; MARKOTIC, D. ; DONG, Y.; FESTIC, E.; KRAJINOVIC, V.; TODOROVIC, Z.; SUSTIC, A.; MILIVOJEVIC, N.; JANDRIC, M.; GAVRILOVIC, S.; NIVEN, A. S.; KOVACEVIC, P.; GAJIC, O. Rapid, multimodal, critical care knowledge-sharing platform for COVID-19 pandemic. Basic Medical Science, v. 21 , n. 1, p-93-97, 2021.

SUNG, E.; GOEBEL, T. International expansion of retailers: the role of technical expertise, alliances and allocation of resources in economic crises.

International Journal of Technology Marketing, v. 13, n. 1, p.1-32, 2019.

TANGARAJA, G.; RASDI, R. M.; SAMAH, B. A.; ISMAIL, M. Knowledge sharing is knowledge transfer: a misconception in the literature. Journal of Knowledge Management, v. 20. n. 4, p. 653-670, 2016.

TAVRA, M.; RACETIN, I.; PEROS, J. The role of crowdsourcing and social media in crisis mapping: a case study of a wildfire reaching Croatian City of Split. Geoenvironmental Disasters, v. 8, p. 1-16, 2021.

TRANFIELD, D.; DENYERD, E.; SMART, P. Towards a methodology for developing evidence-informed management know ledge by means of systematic review. British Journal of Management, v. 14, n. 3, p. 207-222, 2003. 
TSENG, M.-L.; BUI, T.-D.; LIM, M. K. Resource utilization model for sustainable solid waste management in Vietnam: A crisis response hierarchical structure.

Resources, Conservation and Recycling, v. 171, 2021.

TSOURI, M. Knowledge transfer in time of crisis: evidence from the Trentino region. Industry and Innovation, v. 26, n. 7, p. 1-23, 2019.

URICK, M. Generational Differences and COVID-19: Positive Interactions in Virtual Workplaces. Journal of Intergenerational Relationships, v. 18, n. 4, p. 379-398, 2020.

UTUNEN, H.; CHRISTEN, P.; GAMHEWAGE, G. M.; ZHAO, U.; ATTIAS, M. Knowledge transfer for Ebola outbreak - production and use of OpenWHO.org online learning resources. In: INTERNATIONAL CONFERENCE ON WIRELESS AND MOBILE COMPUTING, NETWORKING AND COMMUNICATIONS (WiMob), 14., 2018. Proceedings [...] 2018. p. 20-27,

WENZEL, M.; STANKE, S.; LIEBERMAN, M. B. Strategic responses to crisis. Strategic Management Journal, v. 42, n. 2, p. V7- V18, 2020.

WOLOR, C. W.; AMINAH, H.; MARTONO, S. The Effectiveness of Virtual Work to Keep Achieving Optimal Performance Amid the Covid-19 Virus Outbreak. International Journal of Criminology and Sociology, v. 9, n. 1, p. 310-314, 2020.

ZHANG, Z.; GONZALEZ, M. C.; MORSE, S. S.; VENKATASUBRAMANIAN, V. Knowledge Management Framework for Emerging Infectious Diseases Preparedness and Response: Design and Development of Public Health Document Ontology. JMIR Research Protocols, v. 6. n. 10, 2017.

\title{
KNOWLEDGE SHARING IN CRISIS SITUATIONS: A SYSTEMATIC LITERATURE REVIEW
}

\begin{abstract}
Objective: To present a systematic literature review to discover how to apply knowledge sharing in crisis situations in the last four years. Methodology: Systematic Literature Review conducted by the PRISMA method, with selection of 24 scientific articles, in the period from January 2017 to May 2021. The articles were divided into eight categories: i) trust; ii) institutional memory; iii) diversity in organizations; iv) effects of the crisis on knowledge sharing; v) knowledge sharing tools; vi) techniques and strategies for knowledge sharing; and vii) barriers to knowledge sharing. Results: The results found a heterogeneity of studies as to their characteristics, but verified a predominance in the articles of knowledge sharing in times of crisis referring to techniques and strategies ( $45 \%$ of the total) and in tools for knowledge sharing ( $25 \%$ of the total). In addition, the studies highlighted the importance of using the best tools, practices and strategies for knowledge sharing in crisis situations. Conclusions: The growing importance of this topic in recent years was highlighted due to the COVID-19 pandemic, as well as the need for future studies that can identify the main knowledge sharing strategies to face crisis
\end{abstract}


situations.

Descriptors: Knowledge management. Knowledge sharing. Literature review. Organizational knowledge.

\title{
COMPARTIR CONOCIMIENTOS EN SITUACIONES DE CRISIS: REVISIÓN SISTEMÁTICA DE LA LITERATURA
}

\begin{abstract}
RESUMEN
Objetivo: Presentar una revisión bibliográfica sistemática para descubrir cómo aplicar el intercambio de conocimientos en situaciones de crisis en las organizaciones en los últimos quatro años. Metodología: Revisión sistemática de la literatura realizada por el método PRISMA, con selección de 24 artículos científicos, en el período de enero de 2017 a mayo de 2021. Los artículos se dividieron en ocho categorías: i) confianza; ii) memoria institucional; iii) diversidad en las organizaciones; iv) efectos de la crisis en el intercambio de conocimientos; v) herramientas para el intercambio de conocimientos; vi) técnicas y estrategias para el intercambio de conocimientos; y vii) barreras para el intercambio de conocimientos. Resultados: Los resultados encontraron una heterogeneidad de los estudios en cuanto a sus características, sin embargo se verificó un predominio en los artículos de intercambio de conocimientos en tiempos de crisis referidos a técnicas y estrategias ( $45 \%$ del total) y en las herramientas para el intercambio de conocimientos ( $25 \%$ del total). Además, los estudios destacaron la importancia de utilizar las mejores herramientas, prácticas y estrategias de intercambio de conocimientos en situaciones de crisis. Conclusiones: Se destacó la creciente importancia de este tema en los últimos años debido a la pandemia de COVID-19, y también se verificó la necesidad de futuros estudios que permitan identificar las principales estrategias de intercambio de conocimiento en situaciones de crisis.
\end{abstract}

Descriptores: Conocimiento administrativo. Conocimiento en organizaciones. Intercambio de conocimientos. Revisión de literatura.

Recebido em: 08.09.2021

Aceito em: 17.12 .2021 